\title{
Upper-limb magnetic resonance lymphangiography: a useful new technique
}

\author{
Linfangiografia por ressonância magnética de membros superiores: uma técnica nova e útil
}

\author{
Luís Felipe Fiorentini ${ }^{1, a}$, Guilherme Stüker ${ }^{1, b}$, Gabriel Sartori Pacini $^{2, c}$, Edson Marchiori ${ }^{3, d}$, Bruno Hochhegger ${ }^{4, e}$ \\ 1. Department of Diagnostic Methods, Pontíficia Universidade Católica do Rio Grande do Sul (PUCRS), Porto Alegre, RS, Brazil. 2. Medical Imaging \\ Research Lab (Labimed), Hospital Pavilhão Pereira Filho, Santa Casa de Misericórdia de Porto Alegre, Porto Alegre, RS, Brazil. 3. Universidade \\ Federal do Rio de Janeiro (UFRJ), Rio de Janeiro, RJ, Brazil. 4. Universidade Federal de Ciências da Saúde de Porto Alegre (UFCSPA), Porto Alegre, \\ RS, Brazil. \\ Correspondence: Dr. Bruno Hochhegger. Medical Imaging Research Lab (Labimed), Department of Radiology, Hospital Pavilhão Pereira Filho, \\ Santa Casa de Misericórdia de Porto Alegre. Avenida Independência, 75, Independência. Porto Alegre, RS, Brazil, 90035-074. Email: brunoho@ \\ ufcspa.edu.br. \\ a. https://orcid.org/0000-0002-9315-3692; b. https://orcid.org/0000-0001-9482-0779; c. https://orcid.org/0000-0002-4822-7082; \\ d. https://orcid.org/0000-0001-8797-7380; e. https://orcid.org/0000-0003-1984-4636. \\ Received 2 June 2018. Accepted after revision 10 August 2018.
}

How to cite this article:

Fiorentini LF, Stüker G, Pacini GS, Marchiori E, Hochhegger B. Upper-limb magnetic resonance lymphangiography: a useful new technique. Radiol Bras. 2019 Nov/Dez;52(6):378-379.

\section{INTRODUCTION}

Recent studies have highlighted the importance of magnetic resonance imaging in the evaluation of diseases affecting the musculoskeletal system ${ }^{(1-6)}$. Contrast-enhanced magnetic resonance lymphangiography (MRL) is a recently developed minimally invasive imaging technique for the evaluation of the lymphatic system and its abnormalities. Lymphedema is one of the most widely studied diseases affecting the lymphatic vessels of the upper and lower limbs ${ }^{(7,8)}$; it can be classified as primary (resulting from congenital malformations in vascular morphogenesis, which can be symptomatic later in life) or secondary (mainly resulting from trauma, infection, or malignant tumors).

Novel microsurgical procedures, such as the use of lymphovenous shunts, have allowed major advances to be made in the treatment of lymphedema and lymphatic diseases in general. Simultaneously, the development of new imaging techniques has allowed physicians to identify the anatomical and pathological features of the lymphatic system in order to define the treatment and plan the surgical procedure. In that context, MRL represents an appealing option, because it produces images with high spatial and temporal resolution through the use of three-dimensional enhancement techniques, as well as because, unlike other imaging methods, it does not expose the patient to ionizing radiation $^{(7,8)}$.

Although MRL shows great promise, there are as yet few data available regarding its technical application. In addition, most of the MRL studies conducted to date have focused on the lower limbs, addressing the upper limbs only in a secondary evaluation. Therefore, the objective of the present study was to examine the techniques employed in the upper-limb MRL procedure, demonstrating the main points of the examination and its particularities.

\section{UPPER-LIMB MRL PROCEDURE}

Initially, the patient should be evaluated to determine if there is any contraindication to performing magnetic resonance imaging. Eligible patients should be provided with a full, detailed explanation about the procedure, including its benefits and risks, and written informed consent should be obtained from each patient. After local asepsis, $1-2 \mathrm{~mL}$ of a mixture of contrast media and local anesthetic are injected subcutaneously into each interdigital space in both hands simultaneously. Typically, a mixture of $0.5 \mathrm{~mL}$ $2 \%$ lignocaine and $4.5 \mathrm{~mL}$ of gadobutrol (Gadovist; Bayer Schering Pharma, Berlin, Germany) is administered at a dose of $0.1 \mathrm{mmol} / \mathrm{kg}$ body weight. The injection site is then massaged for $2 \mathrm{~min}$, after which the scan is started. The anesthetic allows the patient to tolerate the procedure, and the contrast media will be continuously drained by the lymphatic system, providing the required information about its integrity/abnormalities. The patient is placed in the prone position, the upper limbs entering the scanner first, allowing the evaluation of the hands, wrists, forearms, elbows, upper arms, and shoulders, in that order. The use of cushions between the coil and the skin can reduce artifacts. Although it is possible to use a $1.5 \mathrm{~T}$ scanner, better results are obtained with a scanner that has a magnetic field strength of 3.0 T or higher ${ }^{(7,8)}$. The MRL protocol included the following: an electrocardiogram-gated steady-state free precession sequence-slice thickness $5 \mathrm{~mm}$, field of view (FOV) $40 \times 40 \mathrm{~mm}$, matrix $256 \times 256$, bandwidth $125 \mathrm{kHz} /$ pixel, and repetition time/echo time (TR/TE) 3.1/1.6 ms; a T1-weighted sequence with spectral inversion at lipidsflip angle $25^{\circ}$, FOV $44 \times 44 \mathrm{~mm}$, matrix $256 \times 180$, slice thickness $3 \mathrm{~mm}$, bandwidth $110 \mathrm{MHz}$, and TR/TE 3.7/2.1 $\mathrm{ms}$; and a 3D T2-weighted turbo spin-echo sequenceFOV $40 \times 40 \mathrm{~mm}$, matrix $320 \times 224$, slice thickness 4 $\mathrm{mm}$, bandwidth $32 \mathrm{MHz}$, and TR/TE 4670/113 ms. As an 

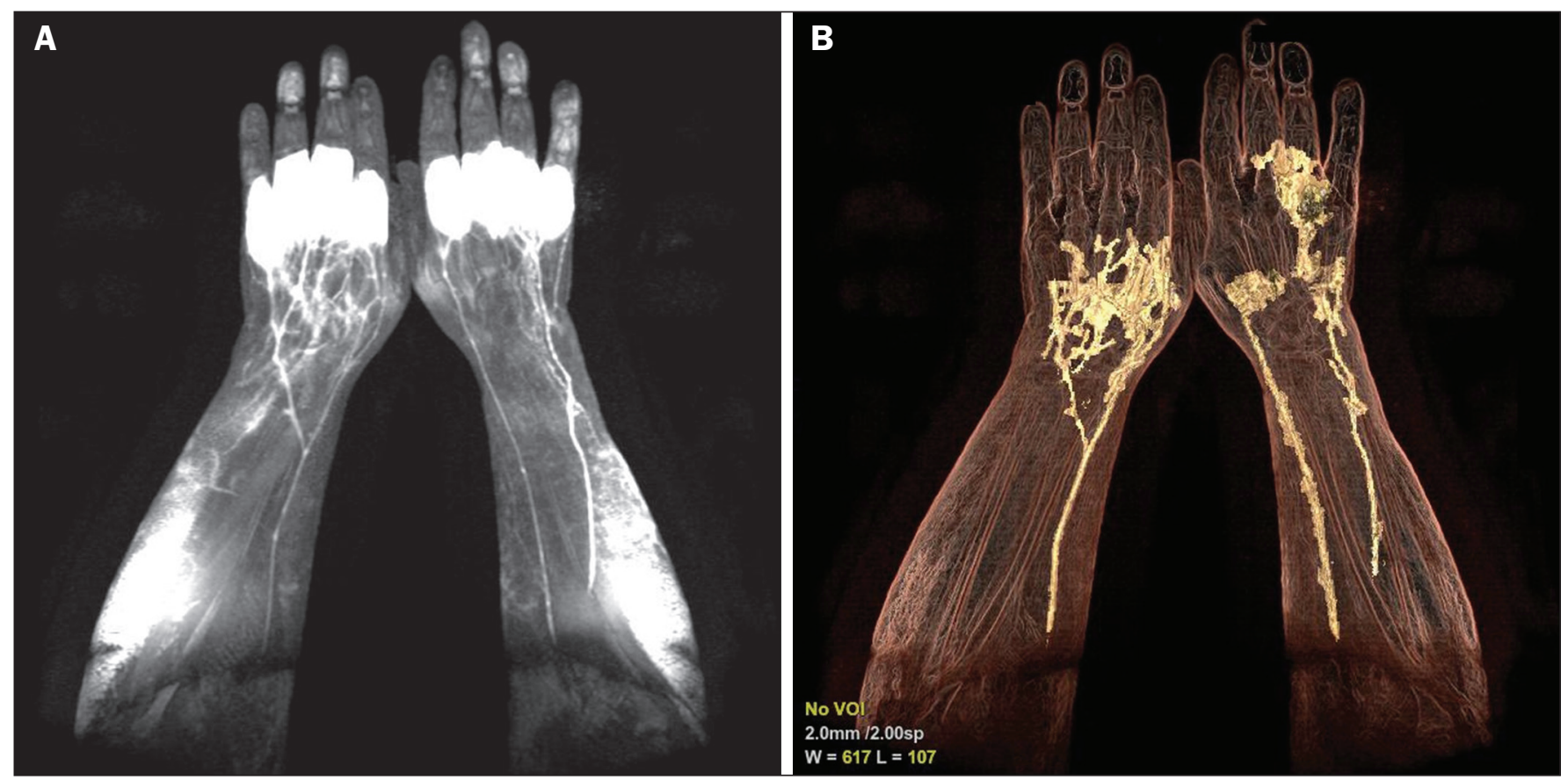

Figure 1. A 54-year-old man with rheumatoid arthritis. A: Maximum intensity projection of a volumetric T1-weighted sequence, with a slice thickness of 1 mm, showing normal lymphatic vessels in both upper limbs, without signs of obstruction. B: The same acquisition with three-dimensional enhancement techniques.

example, we present the MRL imaging obtained in the case of a 54-year-old male patient, previously diagnosed with rheumatoid arthritis, who presented with a 5-year history of lymphedema in the upper limbs (Figure 1).

\section{CONCLUSION}

MRL is a safe, noninvasive imaging method that provides detailed anatomical and functional information regarding the lymphatic system, which is particularly useful in patients with lymphedema. Therefore, it represents a promising tool for the diagnosis and treatment of lymphatic disorders.

\section{REFERENCES}

1. Loures FB, Carrara RJ, Góes RFA, et al. Anthropometric study of the knee in patients with osteoarthritis: intraoperative measurement versus magnetic resonance imaging. Radiol Bras. 2017;50:170-5.

2. Sá Neto JL, Simão MN, Crema MD, et al. Diagnostic performance of magnetic resonance imaging in the assessment of periosteal reactions in bone sarcomas using conventional radiography as the reference. Radiol Bras. 2017;50:176-81.

3. Nascimento PCX, Amaral AM, Almeida JRM. Magnetic resonance arthrography of the shoulder: a painful procedure? Radiol Bras. 2018; 51:81-6.

4. Canella C, Costa F, Danowisk A, et al. Stress fracture and osteomyelitis in a patient with systemic lupus erythematosus. Radiol Bras. 2018;51:277-8.

5. Costa FM, Canella C. Vieira FG, et al. The usefulness of chemicalshift magnetic resonance imaging for the evaluation of osteoid osteoma. Radiol Bras. 2018;51:156-61.

6. Werneck L, Canella C, Costa F, et al. Usefulness of dynamic contrast-enhanced MRI in the evaluation of osteonecrosis of the proximal fragment in scaphoid fractures. Radiol Bras. 2018;51:334.

7. Mitsumori LM, McDonald ES, Neligan PC, et al. Peripheral magnetic resonance lymphangiography: techniques and applications. Tech Vasc Interv Radiol. 2016;19:262-72.

8. Mazzei FG, Gentili F, Guerrini S, et al. MR lymphangiography: a practical guide to perform it and a brief review of the literature from a technical point of view. Biomed Res Int. 2017;2017:2598358. 